\title{
Antecedentes da satisfação no setor público: um estudo de caso na prefeitura de Santa Maria (RS)
}

\author{
Wagner Junior Ladeira \\ Universidade Federal do Rio Grande do Sul \\ Igor Bernardi Sonza \\ Universidade Federal do Rio Grande do Sul \\ Roberto Sarquis Berte \\ Senac Technology School
}

\begin{abstract}
Nos últimos 50 anos, os estudos da satisfação no setor público têm sido objeto de análise de diferentes autores, em diversas partes do mundo, mostrando ser um tema contemporâneo e dinâmico. Com base em uma apreciação das principais publicações nessa área, o presente artigo investigou os antecedentes da satisfação dos funcionários públicos. Na parte teórica/metodológica, avaliaram-se sete construtos (rotinas, especificidade do trabalho, desenvolvimento de recursos humanos, feedback, conflito nas metas organizacionais, restrições processuais e especificidades das metas organizacionais), através da técnica de modelagem de equações estruturais. Foi realizada uma pesquisa descritiva baseada em estudo de caso, sendo aplicados 258 questionários aos funcionários públicos da prefeitura de Santa Maria. O artigo fornece um modelo aplicado com os sete fatores e 11 hipóteses que antecedem a satisfação, encontradas em diversas literaturas da área. Com base nos achados empíricos verifica-se que a satisfação sofre interferência direta de algumas características do trabalho, como: rotinas, especificidades e recursos humanos. E, por consequência, essas características do trabalho recebem influência direta do contexto do trabalho.
\end{abstract}

Palavras-chave: satisfação; modelagem de equações estruturais; características do trabalho; contexto do trabalho.

Antecedents of satisfaction in the public sector: a case study in the city hall of Santa Maria (RS)

In the last fifty years, satisfaction in the public sector has been analyzed by different studies around the world, proving to be a dynamic and contemporary theme. From a review of the main publications in this area, this article investigates the satisfaction background of public employees. In the theoretical/methodological level, we analyze seven constructs (routines, labor specificity, human resource

Artigo recebido em jun. 2010 e aceito em jun. 2011.

RAP - Rio de Janeiro 46(1):71-91, jan./fev. 2012 
development, feedback, organizational goals conflicts, procedural constraints and characteristics of organizational goals), using the structural equation modeling technique. We performed a descriptive study based on case study through the application of 258 questionnaires in civil servants of the city of Santa Maria, RS, Brazil. The study provides an applied model with seven factors and 11 hypotheses that former the satisfaction, anchored in many literatures of the area. With empirical findings support, we found that satisfaction suffers direct interference of some job characteristics, such as routines, specificities and human resources. Consequently, these work characteristics receive direct influence of the work context.

KEY WORDS: satisfaction; structural equation modeling; work characteristic; work context.

\section{Introdução}

Nas últimas décadas o setor público tem vivenciado uma reforma com o objetivo de mudar o seu modus operandi. Nessa reforma, o setor público é cada vez mais um ambiente orientado pelas práticas gerenciais. Isto significa que, em certa medida, o setor público tem incorporado práticas do setor privado. Muitos autores (Roodhooft e Abbeele, 2006; Liu, Tang e Zhu, 2008; Kim, 2006; Brunetto e Farr-Wharton, 2002; Wright e Davis, 2003) descrevem as transformações significativas no setor público, evidenciando o uso de padrões e normas de empreendimentos privados. Essas modificações fizeram com que muitas organizações públicas mudassem a postura com relação a suas metas organizacionais e, para isso, modificaram o contexto e as características do ambiente de trabalho. Atualmente, essas organizações procuram empregar pessoas que são qualificadas e inovadoras, buscando assim atingir metas, atrair, motivar e reter seus funcionários (Bellou, 2009). Essas modificações geraram mudanças no grau de satisfação dos funcionários.

Os estudos de satisfação têm recebido contribuições clássicas (Paine, Carroll e Leete, 1966; Buchanan, 1975; Porter e Mitchell, 1967; Stimson e Johnson, 1977; Perry e Porter, 1982; Daley, 1986) sobre o setor público. Agregando conhecimento a esses estudos clássicos, atualmente muitos autores (Kuvaas, 2009; Bellou, 2009; Addae e Parboteeah, 2008; Liu, Tang e Zhu, 2008; Agus, Barker e Kandampully, 2007; Kim, 2006; Bigné, Moliner e Sánchez, 2003) têm estudado a satisfação através de novas abordagens, dentro do contexto público. Esses estudos ocorrem em diversos países do mundo e têm como característica básica a utilização de práticas empresariais no meio público e sua relação com a satisfação dos funcionários. Além disso, esses estudos são fundamentados em técnicas estatísticas para entender a satisfação dos funcionários do setor público (Andreassen, 1994; Kuvaas, 2009; Bellou, 2009; Addae e Parboteeah, 2008; Roodhooft e Abbeele, 2006; McAdam, Reid e Saulters, 2002; Liu, Tang e Zhu, 2008; Agus, Barker e Kandampully, 2007; Kim, 2006; Fu, Chang e Chao, 2006). Esses estudos ajudam a entender a evolução da satisfação no setor público e são essenciais para aprimorar o conhecimento e, por consequência, obter resultados mais fidedignos a respeito das práticas gerenciais utilizadas.

Com base nesses estudos, que refletem a utilização de práticas gerenciais da iniciativa privada no setor público, o presente artigo visa identificar as relações funcionais da satisfa- 
ção dos funcionários públicos da prefeitura de Santa Maria (RS), tendo como base de análise as variáveis do ambiente de trabalho, que são formadas por dois componentes centrais: características e contexto do trabalho. Este artigo propõe a avaliação de oito construtos que interferem na satisfação dos funcionários públicos. Essa avaliação foi feita através da técnica modelagem de equações estruturais. Para criação desses construtos, o presente artigo revisitou o modelo proposto por Wright e Davis (2003) — desenvolvido no artigo intitulado "Job satisfaction in the public sector: the role of the work environment", publicado na revista The American Review of Public Administration - e utilizou trabalhos clássicos e recentes da literatura do setor público, para dar mais robustez e atualizar os construtos.

\section{Satisfação no setor público: aspectos gerais}

Muitos autores (Baldwin e Farley, 1991; Rainey, 1989; Steel e Warner, 1990; Roodhooft e Abbeele, 2006; Brunetto e Farr-Wharton, 2002; Brewr e Seldon, 2000) estudam o fato de os servidores públicos serem mais ou menos satisfeitos com seu trabalho do que os funcionários do setor privado. Uma suposta causa da insatisfação seria as organizações públicas não oferecerem grandes oportunidades para os servidores desenvolverem suas ideias e satisfazer suas necessidades. Esse conflito entre propósito e estrutura pode ser ilustrado por aparentes inconsistências encontradas em pesquisas na administração pública. Muitos estudos têm mostrado que os servidores públicos são mais satisfeitos (Desantis e Durst, 1996; Maidani, 1991; Steel e Warner, 1990) ou tão satisfeitos quanto (Emmert e Taher, 1992; Gabris, 1995; Lewis, 1991) os trabalhadores do setor privado; outros têm encontrado o oposto, com relação a aspectos específicos de seu trabalho, incluindo o cumprimento de suas responsabilidades, autonomia e atualização (Paine, Carroll e Leete, 1966; Porter e Mitchell, 1967; Solomon, 1986). Mesmo tendo grande quantidade de evidências empíricas das diferenças entre os setores públicos e privados com relação especificamente à satisfação no trabalho, os motivos são variados (Wright, 2001; Babakus et al., 1996). A coerente interpretação desses resultados será o foco dessa pesquisa, preocupando-se mais com a existência do que com as origens dessas diferenças.

\section{Fundamentação das hipóteses}

O ambiente de trabalho é formado por dois componentes: características do trabalho e contexto do trabalho (Wright e Davis, 2003; Desantis e Durst, 1996; Steel e Warner, 1990).

As características do trabalho são os aspectos do trabalho de uma pessoa ou responsabilidades do cargo que contribuem para o seu estado psicológico (Desantis e Durst, 1996; Wright, 2001; Barnett e Bradley, 2007), como a significância do trabalho, que afeta o desenvolvimento, crescimento e disposição dos servidores para o trabalho (Perry e Porter, 1982; Baldwin e Farley, 1991). 
As variáveis do contexto do trabalho são formadas por características organizacionais como sistemas, metas e graus de formalização (Paine, Carroll e Leete, 1966; Solomon, 1986; Kuvaas, 2009). Nas variáveis do contexto espera-se que os servidores desenvolvam seus deveres (Rainey, 1989; Stimson e Johnson, 1977; Bellou, 2009). Em conjunto, as características e o contexto do trabalho representam os fatores externos de satisfação dos servidores e podem ser influenciados e moldados diretamente pelas organizações públicas (Desantis e Durst, 1996; Perry e Porter, 1982; Solomon, 1986; Barnett e Bradley, 2007).

A pesquisa em questão é dividida em oito construtos, sendo quatro deles referentes às características do trabalho (rotina, especificidade do trabalho, desenvolvimento de recursos humanos e feedback), três referentes ao contexto do trabalho (conflito nas metas organizacionais, restrições processuais e especificidade das metas organizacionais) e um diretamente correlacionado à satisfação no trabalho. Estes construtos com as respectivas hipóteses são apresentados na tabela abaixo e descritos no restante desta seção.

Tabela 1

Relacionamento: ambiente de trabalho, construtos e hipóteses

\begin{tabular}{|c|c|c|}
\hline Ambiente de trabalho & Construtos & Hipóteses \\
\hline \multirow[t]{4}{*}{ Características do trabalho } & Rotinas & H1: A rotina tem um efeito direto negativo na satisfação com o trabalho. \\
\hline & $\begin{array}{l}\text { Especificidade do tra- } \\
\text { balho }\end{array}$ & $\begin{array}{l}\text { H2: O grau de especificidade no trabalho tem um efeito direto positivo na } \\
\text { satisfação com o trabalho. }\end{array}$ \\
\hline & $\begin{array}{l}\text { Desenvolvimento de } \\
\text { recursos humanos }\end{array}$ & $\begin{array}{l}\text { H3: A quantidade de oportunidades percebidas no desenvolvimento de recur- } \\
\text { sos humanos tem um efeito direto positivo na satisfação com o trabalho. }\end{array}$ \\
\hline & Feedback & $\begin{array}{l}\text { H4: O nível de feedback que o trabalhador recebe tem um efeito indireto } \\
\text { positivo na satisfação com o trabalho através da influência na especifici- } \\
\text { dade do trabalho. } \\
\text { H5: O nível de feedback tem um efeito indireto positivo na satisfação } \\
\text { através da influência da avaliação das oportunidades de desenvolvimento } \\
\text { de recursos humanos. }\end{array}$ \\
\hline \multirow[t]{3}{*}{ Contexto do trabalho } & $\begin{array}{l}\text { Conflito nas metas } \\
\text { organizacionais }\end{array}$ & $\begin{array}{l}\text { H6: Conflitos nas metas organizacionais têm um efeito diretamente positivo } \\
\text { nas restrições processuais. } \\
\text { H7: Conflitos nas metas organizacionais têm um efeito diretamente negativo } \\
\text { nas metas específicas organizacionais. }\end{array}$ \\
\hline & $\begin{array}{l}\text { Restrições processu- } \\
\text { ais }\end{array}$ & $\begin{array}{l}\text { H8: Restrições processuais têm um efeito adverso na satisfação do em- } \\
\text { pregado, que se expressa através das rotinas do cargo. } \\
\text { H9: Restrições processuais têm um efeito adverso na satisfação do empre- } \\
\text { gado, que se expressa através do grau de especificidade de seu cargo. }\end{array}$ \\
\hline & $\begin{array}{l}\text { Especificidades das } \\
\text { metas organizacio- } \\
\text { nais }\end{array}$ & $\begin{array}{l}\text { H10: As metas específicas organizacionais têm um efeito positivo direto na } \\
\text { satisfação do funcionário, dependendo do grau de feedback que recebe } \\
\text { do RH e das especificidades de seu cargo. } \\
\text { H1 1: As metas específicas organizacionais têm um efeito positivo direto na } \\
\text { satisfação do funcionário, dependendo da influência do RH em seu cargo. }\end{array}$ \\
\hline
\end{tabular}




\subsection{Características do trabalho no setor público}

Várias ligações entre características do trabalho e satisfação têm sido exploradas na literatura, geralmente comparando a satisfação no trabalho entre trabalhadores dos setores públicos e privados (Wright e Davis, 2003; Desantis e Durst, 1996; Ting 1996; Lewis e Alonso, 2001).

As características do trabalho podem ser entendidas como "o que as pessoas fazem no trabalho, ou seja, a natureza do trabalho em relação às tarefas que são compreendidas” (Perry e Porter, 1982), e podem ser consideradas uma das principais determinantes da satisfação no trabalho. O interesse particular nas características do trabalho se dá pelo fato de elas representarem antecedentes da satisfação no trabalho. Das diversas características do trabalho, quatro em especial são estudadas nesta pesquisa. Três têm um efeito direto no trabalho: rotina, especificidade e recursos humanos. Da quarta característica do trabalho, o feedback, espera-se que tenha um efeito indireto na satisfação, pois é decorrente do efeito dos recursos humanos e da especificidade do trabalho (Sherman e Bohlander, 1992; Addae e Parboteeah, 2008). As características do trabalho, e sua relação com a satisfação, são explanadas a seguir.

\subsubsection{Rotinas}

Como uma característica do trabalho, a rotina é o grau de previsibilidade com que o servidor se defronta no dia a dia. Em outras palavras, são as tarefas diárias repetitivas que não necessitam de variedade de experiências e habilidades. Pesquisas como a de Stimson e Johnson (1977) sugerem que trabalhadores — que possuem uma variedade de experiências e tarefas e usam suas habilidades com frequência - têm experiências menos tediosas e, por consequência, mais satisfação no trabalho. Quando os servidores percebem que o trabalho se torna mais rotineiro, o nível de satisfação diminui. Consistente com essa questão, é esperado que: hipótese 1 - a rotina tem um efeito direto negativo na satisfação com o trabalho.

\subsubsection{Especificidade do trabalho}

A especificidade no trabalho faz com que os deveres sejam claramente transmitidos. Desse modo, o servidor pode avaliar seu sucesso e seu fracasso na performance das tarefas. Pesquisas como as de Daley (1986) e Ting (1996) identificaram como um efeito positivo que a especificidade no trabalho leva à satisfação. Quando o servidor entende mais claramente o que seus superiores esperam dele, tensões associadas à ambiguidade decrescem e a probabilidade de sucesso em suas responsabilidades aumenta, trazendo maior satisfação. Então, é esperado que: hipótese 2 - o grau de especificidade no trabalho tem um efeito direto positivo na satisfação com o trabalho. 


\subsubsection{Recursos humanos}

Recursos humanos (RH) referem-se às percepções sobre oportunidades de treinamento, ascensão profissional e desenvolvimento das habilidades na organização. Os programas de $\mathrm{RH}$ tendem a aumentar a produtividade e, consequentemente, a probabilidade de sucesso nas tarefas dos servidores (Sherman e Bohlander, 1992; Roodhooft e Abbeele, 2006). Logo, além de aumentar a produtividade organizacional e a performance de seu trabalho (Budd e Broad, 1996), os programas de RH trabalham com questões importantes que influenciam a satisfação no trabalho, reduzindo o estresse ou a insatisfação no trabalho. Então, é esperado que: hipótese 3 - a quantidade de oportunidades percebidas no desenvolvimento de recursos humanos tem um efeito direto positivo na satisfação com o trabalho.

\subsubsection{Feedback}

Trabalhadores recebem feedback de sua performance individual de seus supervisores, colegas de trabalho e clientes. Em uma perspectiva organizacional, espera-se que este feedback ocorra periodicamente ou por meio de avaliações de performance, ajudando a definir a expectativa com relação ao trabalho (Kuvaas, 2009; Andreassen, 1994). Este feedback não só promove um mecanismo que guia a ação, mas também desenvolve habilidades necessárias ou julgamentos específicos do trabalho (Addae e Parboteeah, 2008; Barnett e Bradley, 2007). Como resultado, o feedback pode ter também a função de definir a relação entre o trabalhador e a organização, indicando as possibilidades correntes de alcançar os objetivos da organização, tanto quanto identificar potenciais que o trabalhador pode desenvolver no futuro. De acordo com esse pressuposto, duas hipóteses foram identificadas: hipótese 4 - o nível de feedback que o trabalhador recebe tem um efeito indireto positivo na satisfação com o trabalho através da influência na especificidade do trabalho; hipótese 5 - o nível de feedback tem um efeito indireto positivo na satisfação através da influência da avaliação das oportunidades de desenvolvimento de recursos humanos.

\subsection{Contexto do trabalho no setor público}

Em contraste com as características do trabalho, que têm efeito imediato e direto no desenvolvimento das tarefas do servidor, o contexto do trabalho se refere às características do ambiente, metas e grau de formalização organizacional em que o servidor desenvolve seu trabalho. Embora fatores do contexto organizacional normalmente busquem a relação existente entre a organização e a eficiência, é esperada uma relação entre as condições de trabalho e as atitudes e os comportamentos dos servidores (Barnett e Bradley, 2007; Snyder, Osland e Hunter, 1996). 
O contexto do trabalho em uma organização, através de sua estrutura e objetivos, poderá não exercer uma influência direta na atitude e no comportamento dos funcionários, mas em muitos casos observa-se uma influência indireta (Addae e Parboteeah, 2008; Wright e Davis, 2003). Esta influência pode advir de três aspectos determinantes: conflito nas metas ou nos objetivos organizacionais, restrições pessoais e especificidade das metas organizacionais.

\subsubsection{Conflito nas metas organizacionais}

As metas organizacionais no setor público são objetos de estudo que vêm sendo analisados por diversos autores (Bellou, 2009; Barnett e Bradley, 2007), principalmente quando o foco da análise são os conflitos que seguem essas metas (Behn, 1995; Wright e Davis, 2003). A escassez de planejamento das instituições públicas pode acarretar uma má interpretação das metas organizacionais, que em muitos casos pode levar a conflitos. Aliando-se à escassez de planejamento, muitas organizações públicas recebem influência de diversos agentes e, por isso, são frequentemente vistas como um ente com metas organizacionais conflitantes. Tais conflitos geram imprecisões nas tarefas diárias dos empregados (Behn, 1995; Bellou, 2009; Kuvaas, 2009). É mais fácil identificar o que os empregados não fazem do que aquilo que eles deveriam fazer (Behn, 1995). Desse modo, os conflitos nas metas organizacionais podem gerar restrições processuais, deixando o trabalho mais burocrático. Por exemplo, se o funcionário não souber as metas organizacionais, isso pode acarretar confusões de prioridades em seu serviço, gerando problemas também nas metas gerais da organização. Portanto, de acordo com esse pressuposto, duas hipóteses foram identificadas: hipótese 6 - conflitos nas metas organizacionais têm um efeito direto negativo nas restrições processuais; hipótese 7 - conflitos nas metas organizacionais têm um efeito direto negativo nas metas específicas organizacionais.

\subsubsection{Restrições processuais}

Há uma relação de dependência entre as regras organizacionais e as expectativas dos funcionários. As restrições processuais refletem o estado no qual os funcionários estão coagidos pelas regras organizacionais (Buchanan, 1975; Kim, 2006). Os antecedentes das restrições processuais podem influenciar diretamente a satisfação em relação ao cargo dos funcionários. Quando as restrições processuais são grandes dentro das organizações públicas, acabam inibindo a criatividade dos funcionários, fazendo com que eles interpretem suas atividades através de rotinas cansativas. As restrições processuais podem também interferir diretamente no grau de especificidade dos cargos. Quanto maior o número de restrições processuais maior será o grau de especificidade dos cargos (Meyer, 1982; Bellou, 2009; Wright e Davis, 2003). Portanto, de acordo com esse pressuposto, duas hipóteses foram identificadas: hipótese 8

— restrições processuais têm um efeito adverso na satisfação do empregado, que se expressa 
através das rotinas do cargo; hipótese 9 - restrições processuais têm um efeito adverso na satisfação do empregado, que se expressa através do grau de especificidade de seu cargo.

\subsubsection{Especificidades das metas organizacionais}

O conteúdo das metas de trabalho está envolvido diretamente com o comprometimento do empregado. O conteúdo de uma meta pode ser entendido como a especificidade na descrição das naturezas da tarefa que compõem o trabalho. Isso se reflete no comprometimento dos trabalhadores, de suas atitudes e crenças em relação ao trabalho. A especificidade das metas organizacionais representa o grau de compreensão dos objetivos organizacionais por parte dos funcionários (Wright, 2001; Jamali, 2007; Wright e Davis, 2003). Semelhantemente às restrições processuais, as especificidades das metas organizacionais podem ser consideradas um antecedente que leva à satisfação. Conhecimento claro das metas organizacionais pode levar a um conhecimento maior das especificidades do próprio cargo. Da mesma forma, o conhecimento claro das especificidades das metas organizacionais, provavelmente, poderá levar a conhecimentos claros das estratégias da organização e, por conseguinte, influenciar diretamente a satisfação (Kuvaas, 2009; Kim, 2006). Portanto, de acordo com esse pressuposto, duas hipóteses foram identificadas: hipótese 10 - as metas específicas organizacionais têm um efeito positivo direto na satisfação do funcionário, dependendo do grau de feedback que recebe do $\mathrm{RH}$ e da especificidade de seu cargo; hipótese 11 - as metas específicas organizacionais têm um efeito positivo direto na satisfação do funcionário, dependendo da influência do RH em seu cargo.

\section{Aspectos metodológicos}

Para o desenvolvimento do presente artigo foi utilizada uma pesquisa descritiva baseada em estudo de caso. Nesse sentido, Gil (2002) destaca que este tipo de pesquisa tem como objetivo primordial a descrição sobre determinada população ou fenômeno, ou então o estabelecimento de relações entre variáveis. Já para Hair e colaboradores (2006), a pesquisa descritiva mede as características descritas em uma questão de pesquisa.

Os dados primários foram coletados através de questionários estruturados adaptados da modelagem criada por Wright e Davis (2003). As perguntas foram desenvolvidas em uma escala de 1 (discordo totalmente) a 6 (concordo totalmente). Foram utilizados, na elaboração deste artigo, dados secundários extraídos: i) da internet; ii) de pesquisa bibliográfica; iii) de pesquisa documental. Os questionários foram respondidos aleatoriamente pelos servidores públicos da Prefeitura Municipal de Santa Maria, Rio Grande do Sul, representando uma amostra total de 258 respondentes, atingindo todas as secretarias. Foi aplicado um pré-teste para três servidores, que comprovaram a eficiência, a fim de que os mesmos projetassem suas próprias concepções e valores a respeito do tema proposto. 
Para a realização do estudo, foi aplicado o método de equações estruturais, como evidenciado na figura 1. Essa técnica examina uma série de relações de dependência simultaneamente entre as variáveis (Hair et al., 2006). O modelo estrutural especifica as relações entre as variáveis e descreve a quantidade de variância explicada (Schumacker e Lomax, 1996). Para identificar a confiabilidade, ou seja, o grau de consistência interna entre os múltiplos indicadores de um construto, referindo-se à extensão na qual um mesmo instrumento de medida produz resultados coerentes a partir de diversas mensurações, é necessário que o índice de confiabilidade seja maior que 0,7 (Schumacher e Lomax, 1996). Se os resíduos padronizados dos indicadores de um construto são altos, podem estar medindo mais de um construto e podem não ser unidimensionais. Para um nível de significância de 5\%, são considerados altos os resíduos padronizados superiores a 2,58 (Garver e Mentzer, 1999).

Para testar a validade da equação estrutural a ser formada, foram utilizadas as medidas descritas a seguir: i) $\chi^{2}$ (qui-quadrado) — avalia a significância das diferenças entre a matriz observada e a matriz estimada (Hair et al., 1999); os níveis de significância estatística indicam a probabilidade de que a diferença seja devida à variação amostral; o pesquisador está interessado em obter um valor de $\chi^{2}$ não significativo, indicando que os dados se ajustam ao modelo (Schumacker e Lomax, 1996); ii) Root mean square residual (RMSR) — é a raiz quadrada dos resíduos ao quadrado (Hair et al., 1999); quando o ajuste do modelo é perfeito a RMSR é igual a zero; quando a discrepância média entre as covariâncias observadas e preditas aumenta, o índice também aumenta; Kline (1998) sugere que um valor menor do que 0,10 é aceitável; iii) Root mean square error of aproximation (RMSEA) — representa a discrepância entre as matrizes observadas e previstas, levando em consideração os graus de liberdade; valores maiores que 0,5 são considerados aceitáveis (Hair et al., 1999); iv) Goodness-of-fit (GFI) - considera a quantidade de variância e covariância da matriz observada que é reproduzida pela matriz estimada (Schumacker e Lomax, 1996); os valores geralmente variam de zero (ajuste ruim) a um (ajuste perfeito), mas pode haver valores negativos ou maiores do que um quando a amostra é pequena ou quando o modelo é over-identificado (Kline, 1998); v) Comparative fit index (CFI) - medida comparativa global entre os modelos estimado e nulo, seu valor indica a proporção das covariâncias observadas que podem ser explicadas pelo modelo; valores superiores a 0,9 seriam desejáveis (Kline, 1998); vi) Normed fit index (NFI) — indica a proporção em que o ajuste do modelo proposto é melhor do que o ajuste do modelo nulo; segundo Hair et al. (1999), não existe um valor absoluto que indique um nível de ajuste aceitável, mas recomenda-se que seja superior a 0,9; vii) Tucker-Lewis index ou non-normed fit index (NNFI) - é interpretado da mesma maneira que o NFI, no entanto, inclui um ajuste para a complexidade do modelo; especificamente, modelos mais complexos, aqueles com maior número de parâmetros, tendem a apresentar ajustes melhores do que os modelos mais simples, para uma mesma amostra (Kline, 1998); seus valores geralmente variam de zero a um e recomenda-se um nível superior a 0,9 (Pedhazur e Schmelkin, 1991; Hair et al., 1999; Kline, 1998). 
Figura 1

Hipóteses do modelo

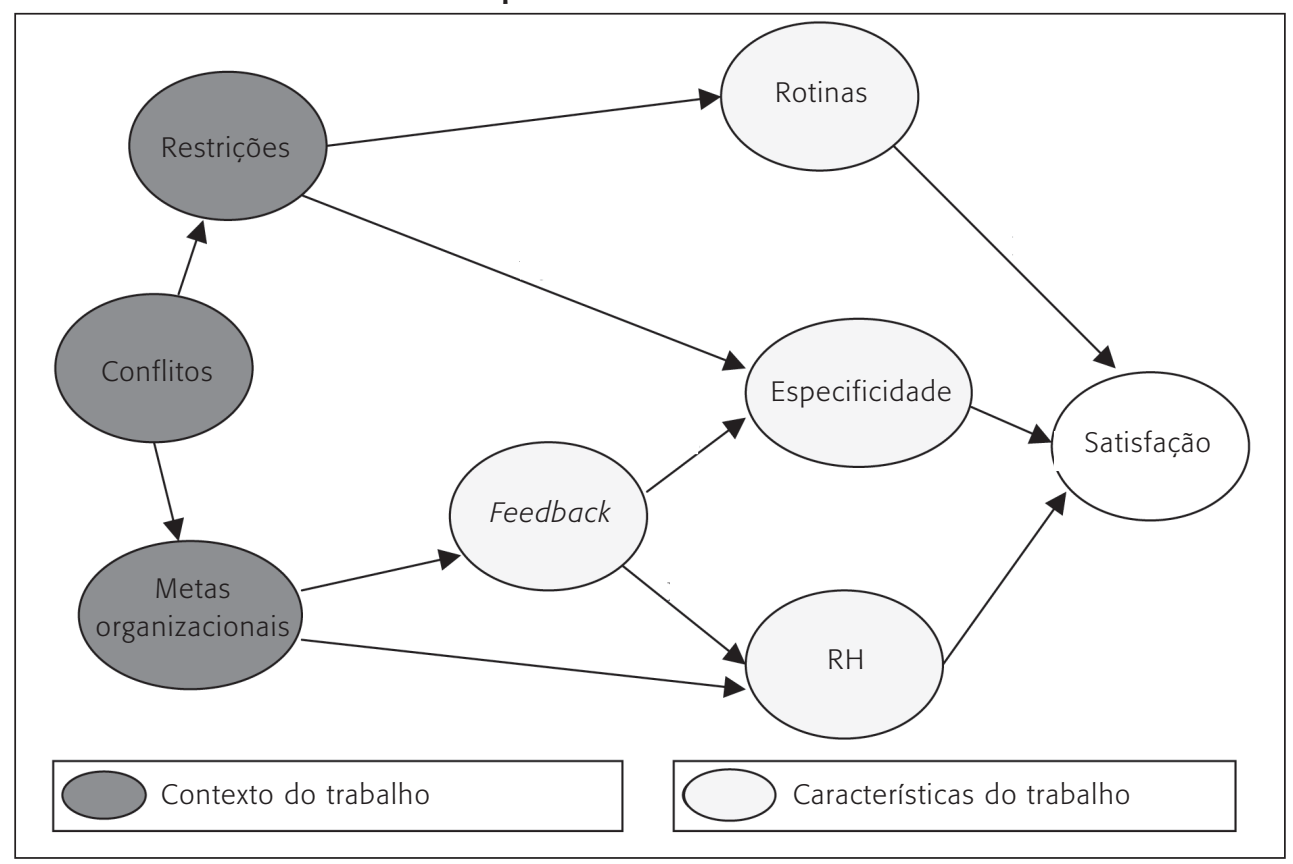

Foram utilizados dois métodos para testar a confiabilidade dos dados, como descrito a seguir: i) índice de confiabilidade maior que 0,7 ; ii) variância extraída maior que 0,5. Caso estes índices não sejam satisfatórios, pode ser utilizado o método de análise de confiabilidade Alpha de Cronbach, que segundo Siegle (2004) serve para avaliar a confiabilidade de uma avaliação que sumaria um grupo do teste. Nesse teste, Nunnaly (1978) indica 0,7 como um coeficiente aceitável da confiabilidade, mas outros autores consideram pontos iniciais mais baixos. A seguir é apresentada a análise dos resultados do estudo.

\section{Análise de resultados}

Como foi descrito na revisão bibliográfica, a satisfação no setor público pode ter como antecedentes: rotinas, restrições, conflitos, especificidades, metas organizacionais, feedback e RH. Dentro desse contexto, o estudo em questão analisou a satisfação com base em sete construtos. Para melhor explicitar os resultados obtidos, este capítulo foi dividido em três partes: i) estatística descritiva das variáveis; ii) validação individual dos construtos; iii) análise do modelo integrado. 


\subsection{Estatística descritiva}

Através da análise estatística descritiva dos 258 questionários respondidos, foi identificado que grande parte dos pesquisados encontra-se acima dos 30 anos - por volta de $84 \%$ - e que grande parte é do sexo masculino - 71,7\%. Com relação ao cargo que ocupam, foram entrevistados trabalhadores de 31 tipos diferentes de funções exercidas, nas 13 secretarias existentes. Esses dados evidenciam o perfil do público analisado.

Com relação ao sexo dos respondentes, foi comprovado que não existe correlação significativa entre essa variável, o cargo ocupado e a respectiva secretaria, pois foi aceita a hipótese nula (H0) devido a este índice ser maior que 0,05 . Já com relação à idade, percebe-se que há uma diferença significativa entre esta e o cargo exercido ou a secretaria a que pertence, pois o teste qui-quadrado mostrou-se representativo, já que este índice apresentou valor inferior a 0,05 .

\subsection{Validação individual dos construtos}

Partindo do modelo apresentado na metodologia, construtos seriam formados por três, quatro ou cinco variáveis. Os coeficientes estimados oferecem informações sobre a extensão na qual uma dada variável observável é capaz de medir uma variável latente (Schumacker e Lomax, 1996). Observa-se que os coeficientes estimados apresentam valores positivos e significativos, evidenciando que os indicadores estão positivamente relacionados com o construto. No entanto, os índices de ajuste do modelo não foram adequados na análise inicial.

Os valores finais dos coeficientes padronizados dos construtos são apresentados no quadro 1 . Pode-se perceber que quase todos os coeficientes possuem índice menor que 0,5 , indicando que todos eles são significativos para o modelo. Apenas um coeficiente padronizado, o da variável 42, teve nível de significância acima de 90\%. A variável 21 teve nível de significância de 0,284 , observando desse modo a significância baixa da variável.

Quadro 1

Coeficientes padronizados e significância dos construtos

\begin{tabular}{|lclcrcrrr|}
\hline Variáveis & Relações & Construto & Est. não Pad. & Est. Pad. & DP & Z & p-valor & Sig. \\
\hline Restrições & $<--$ & Conflitos & $-0,523$ & $-0,72$ & 0,081 & $-6,474$ & 0 & $* * *$ \\
Rotinas & $<--$ & Restrições & $-0,454$ & $-0,785$ & 0,101 & $-4,473$ & 0 & $* * *$ \\
Metas org. & $<--$ & Conflitos & $-0,736$ & $-0,783$ & 0,083 & $-8,885$ & 0 & $* * *$ \\
Satisfação & $<-$ & Especificidade & 1,097 & 0,565 & 0,288 & 3,805 & 0 & $* * *$ \\
Satisfação & $<-$ & Rotinas & $-0,379$ & $-0,236$ & 0,174 & $-2,179$ & 0,029 & $* *$ \\
Feedback & $<--$ & Metas org. & 0,723 & 0,657 & 0,083 & 8,746 & 0 & $* * *$ \\
Satisfação & $<--$ & RH & 0,223 & 0,32 & 0,071 & 3,148 & 0,002 & $* * *$ \\
\hline
\end{tabular}

Continua 


\begin{tabular}{|c|c|c|c|c|c|c|c|c|}
\hline Variáveis & Relações & Construto & Est. não Pad. & Est. Pad. & DP & Z & p-valor & Sig. \\
\hline Especificidade & $<->$ & $\mathrm{RH}$ & 0,317 & 0,513 & 0,073 & 4,344 & 0 & $* * *$ \\
\hline Conflitos & $<->$ & Especificidade & $-0,338$ & $-0,528$ & 0,076 & $-4,446$ & 0 & $* * *$ \\
\hline Conflitos & $<->$ & $\mathrm{RH}$ & $-1,392$ & $-0,78$ & 0,197 & $-7,085$ & 0 & $* * *$ \\
\hline V61 & $<-$ & Restrições & 1 & 0,596 & & & & \\
\hline V62 & $<-$ & Restrições & $-0,455$ & $-0,302$ & 0,127 & $-3,576$ & 0 & $* * *$ \\
\hline V63 & $<-$ & Restrições & 0,213 & 0,164 & 0,102 & 2,081 & 0,037 & $* *$ \\
\hline V64 & $<-$ & Restrições & $-0,738$ & $-0,467$ & 0,161 & $-4,578$ & 0 & $* * *$ \\
\hline V11 & $<-$ & Satisfação & 1 & 0,734 & & & & \\
\hline V12 & $<-$ & Satisfação & 1,16 & 0,733 & 0,113 & 10,224 & 0 & $* * *$ \\
\hline V13 & $<-$ & Satisfação & 0,543 & 0,396 & 0,101 & 5,36 & 0 & $* * *$ \\
\hline V14 & $<-$ & Satisfação & 0,829 & 0,452 & 0,137 & 6,061 & 0 & $* * *$ \\
\hline V24 & $<-$ & Especificidade & 1 & 0,479 & & & & \\
\hline V23 & $<-$ & Especificidade & 1,585 & 0,51 & 0,341 & 4,644 & 0 & $* * *$ \\
\hline V22 & $<-$ & Especificidade & $-0,249$ & $-0,085$ & 0,232 & $-1,07$ & 0,284 & \\
\hline V21 & $<-$ & Especificidade & 2,034 & 0,738 & 0,337 & 6,044 & 0 & $* * *$ \\
\hline V34 & $<-$ & Feedback & 1 & 0,812 & & & & \\
\hline V33 & $<-$ & Feedback & 0,722 & 0,641 & 0,078 & 9,212 & 0 & $* * *$ \\
\hline V32 & $<-$ & Feedback & 0,728 & 0,663 & 0,08 & 9,071 & 0 & $* * *$ \\
\hline V31 & $<-$ & Feedback & 0,704 & 0,624 & 0,08 & 8,844 & 0 & $* * *$ \\
\hline V41 & $<-$ & Rotinas & 1 & 0,41 & & & & \\
\hline V42 & $<-$ & Rotinas & 0,37 & 0,149 & 0,231 & 1,604 & 0,1 & $*$ \\
\hline V43 & $<-$ & Rotinas & 2,28 & 0,764 & 0,467 & 4,88 & 0 & $* * *$ \\
\hline V44 & $<-$ & Rotinas & 0,644 & 0,235 & 0,249 & 2,586 & 0,01 & $* * *$ \\
\hline V53 & $<-$ & $\mathrm{RH}$ & 1 & 0,772 & & & & \\
\hline V52 & $<-$ & $\mathrm{RH}$ & $-0,418$ & $-0,353$ & 0,084 & $-4,989$ & 0 & $* * *$ \\
\hline V51 & $<-$ & $\mathrm{RH}$ & 1,094 & 0,854 & 0,089 & 12,297 & 0 & $* * *$ \\
\hline V65 & $<-$ & Restrições & $-0,636$ & $-0,36$ & 0,149 & $-4,265$ & 0 & $* * *$ \\
\hline V71 & $<-$ & Conflitos & 1 & 0,722 & & & & \\
\hline V72 & $<-$ & Conflitos & 0,845 & 0,713 & 0,08 & 10,493 & 0 & $* * *$ \\
\hline V73 & $<-$ & Conflitos & 0,956 & 0,772 & 0,084 & 11,313 & 0 & $* * *$ \\
\hline V74 & $<-$ & Conflitos & 0,758 & 0,614 & 0,086 & 8,82 & 0 & $* * *$ \\
\hline V83 & $<-$ & Metas org. & 1 & 0,829 & & & & \\
\hline V82 & $<-$ & Metas org. & 1,083 & 0,889 & 0,068 & 15,872 & 0 & $* * *$ \\
\hline V81 & $<-$ & Metas org. & 0,909 & 0,723 & 0,075 & 12,112 & 0 & $* * *$ \\
\hline
\end{tabular}

* Nível de significância de 90\%; **Nível de significância de 95\%; ***Nível de significância de 99\%. 


\subsection{Análise do modelo integrado}

Após a aplicação da análise fatorial confirmatória para a construção e validação dos construtos, buscou-se avaliar o modelo integrado que agrega o modelo de mensuração e o modelo estrutural. Nessa etapa, o objetivo principal era avaliar a estrutura teórica da hipótese, ou seja, as relações entre os construtos e as variáveis propostas no modelo.

A hipótese 1, que corresponde ao inter-relacionamento entre os construtos rotinas e satisfação, que indica que "a rotina tem um efeito direto negativo na satisfação com o trabalho", foi validada tendo como relação o valor de -2,4. Com relação à hipótese 2 , que mede o relacionamento entre os construtos especificidade e satisfação, notou-se que esta é validada, pois seu valor foi de 0,56 , caracterizando o fato que "o grau de especificidade no trabalho tem um efeito direto positivo na satisfação com o trabalho". A hipótese 3, que se refere ao construto de recursos humanos, foi validada com um valor de 0,32 , demonstrando que a "quantidade de oportunidades percebidas no desenvolvimento de RH tem um efeito direto positivo na satisfação com o trabalho".

O construto feedback propunha avaliar duas hipóteses. E nenhuma das duas hipóteses foi representada no modelo novo. A hipótese 4 - "o nível de feedback que o trabalhador recebe tem um efeito indireto positivo na satisfação com o trabalho através da influência na especificidade do trabalho" - e a hipótese 5 - "o nível de feedback tem um efeito indireto positivo na satisfação através da influência da avaliação das oportunidades de desenvolvimento de recursos humanos" - não foram consideradas dentro do novo modelo e foram descartadas para estimações de novas relações.

No que tange ao construto conflitos, as duas hipóteses foram validadas. Observou-se no novo modelo que a relação da hipótese 6 - "conflitos nas metas organizacionais têm um efeito direto negativo nas restrições processuais" - foi verificada no modelo, com um valor de -0,72. Já a hipótese 7 - "conflitos nas metas organizacionais têm um efeito diretamente negativo nas metas específicas organizacionais" - teve um valor de $-7,8$, demonstrando ser válida dentro do novo modelo estimado.

Com relação ao construto restrições, de forma semelhante ao ocorrido nas duas hipóteses ligadas ao construto conflitos, a hipótese 8 foi validada, tendo uma relação de $-7,8$, o que significa que "restrições processuais têm um efeito adverso na satisfação do empregado, que se expressa através das rotinas do cargo". No entanto, foi sugerido no novo modelo desconsiderar a hipótese 9 - "restrições processuais têm um efeito adverso na satisfação do empregado, que se expressa através do grau de especificidade de seu cargo".

Com respeito às hipóteses ligadas ao construto metas organizacionais, as duas tiveram comportamentos diferentes. No caso da hipótese 10 - "as metas específicas organizacionais têm um efeito positivo direto na satisfação do funcionário, dependendo do grau de feedback que recebe do RH e da especificidade de seu cargo" - , ela foi validada, com um valor de 0,66. Já a hipótese 11 - "as metas específicas organizacionais têm um efeito positivo direto na satisfação do funcionário, dependendo da influência do RH em seu cargo" - não obteve uma relação dentro do novo modelo. 
Constatando a validação de algumas hipóteses (H1, H2, H3, H6, H7, H8, H9 e H10) e a retirada de outras (H4, H5 e H11), novas relações geraram modificações no modelo original. Com base nas modificações realizadas no modelo, seguindo recomendação de diversos autores (Kline, 1998; Hair et al., 1999, entre outros), a avaliação do modelo teórico foi realizada a partir dos índices de ajuste do modelo e da significância estatística dos coeficientes de regressão estimados. Nessa fase optou-se pela estratégia de aprimoramento do modelo. Cabe ressaltar que na modificação do modelo proposto foram sendo retirados os coeficientes de regressão não significativos e incorporadas covariâncias não previstas inicialmente. As novas relações, mesmo que sugeridas pelo relatório de modificações do $\mathrm{AMOS}^{\mathrm{TM}}$, só foram adicionadas quando apresentaram uma argumentação teórica que as justificasse.

Os índices de ajustamento do modelo proposto não foram satisfatórios. O qui-quadrado é significativo, provavelmente pela sensibilidade do teste em relação ao tamanho da amostra. Mesmo a relação qui-quadrado/graus de liberdade apresentou um valor de 4,24, acima do limite de 3 recomendado por Kline (1998). Nenhum índice de ajuste estava dentro do limite recomendado. Além disso, observa-se que vários coeficientes não são significativos.

Diante de tais resultados, adotou-se a estratégia de aprimoramento do modelo. Tal estratégia consistiu basicamente da retirada das relações não significativas e da avaliação das modificações sugeridas pelo AMOS $^{\mathrm{TM}}$, que nesse caso incluíram novas relações. O processo de retirada envolveu a eliminação individual de cada uma das relações não significativas já que, a cada retirada, havia necessidade de reestimação do modelo, devido às modificações nos coeficientes e significâncias das demais variáveis. Para o ajuste do construto, além das variáveis retiradas na análise fatorial, foi necessária a realocação dos relacionamentos entre os construtos. Este procedimento está de acordo com a proposição de que devem ser retiradas as relações cujos coeficientes estejam entre os menores ou não sejam significativos, ou seja, que obtiverem valores menores que 0,5 (Kline, 1998).

Verificando as modificações sugeridas pelo AMOS $^{\mathrm{TM}}$, julgou-se conveniente a adoção das seguintes covariâncias entre os construtos, além das realizadas na análise dos construtos individuais:

v conflitos e especificidade - encontram-se intimamente ligados, com uma relação inversa de -0,53. Esta relação demonstra que os conflitos nas metas geram imprecisões nas tarefas diárias dos empregados e, por consequência, interferem negativamente no entendimento do que seus superiores querem dizer; o inverso também é verdadeiro, ou seja, especificidade influencia os conflitos negativamente;

v especificidade e recursos humanos - o grau de especificidade no trabalho e os recursos humanos têm um efeito recíproco de 0,51 ; portanto, os erros destas medidas estão relacionados. Isto demonstra que o funcionário entende mais claramente o que seus superiores esperam dele, tensões associadas com a ambiguidade decrescem e a probabilidade de sucesso em suas responsabilidades aumenta, trazendo maior relação com a possibilidade de oportunidades de treinamento, ascensão profissional e desenvolvimento das habilidades na organização, e vice-versa; 
v conflitos e recursos humanos - os conflitos nas metas organizacionais podem gerar menor probabilidade de ocorrer ascensão profissional e desenvolvimento das habilidades na organização; o relacionamento aqui identificado é de 0,56 , tanto na direção conflitos para recursos humanos, como na contrária.

Figura 2

\section{Modelo integrado}

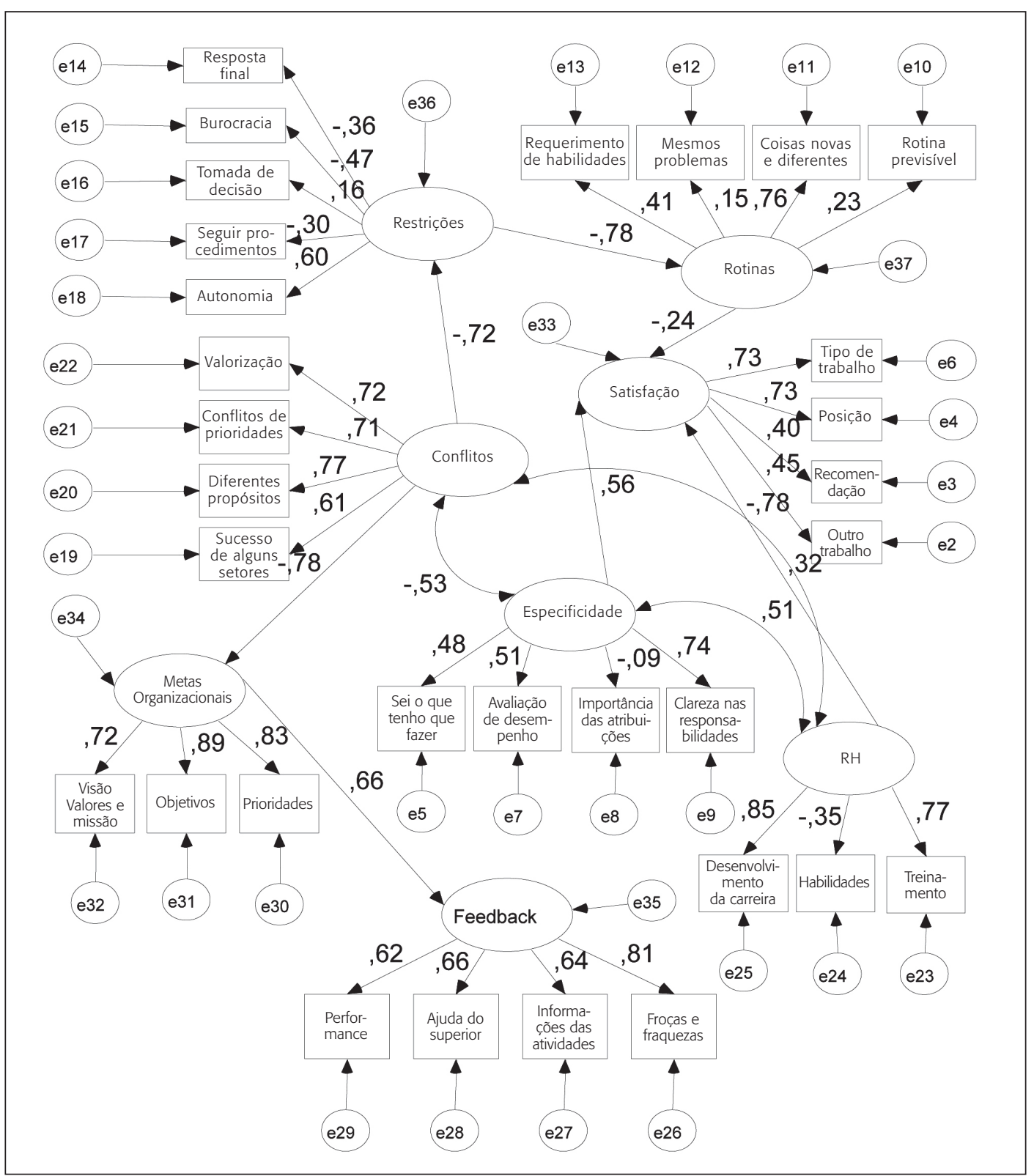


Após a retirada das relações não significativas e da inserção das covariâncias sugeridas, o modelo final apresentou os índices de ajuste descritos no quadro 2 . O desvio padrão e o teste $\mathrm{Z}$ foram significativos em ambos os construtos. O modelo integrado de satisfação dos funcionários públicos da prefeitura de Santa Maria (RS), levando em consideração as variáveis do ambiente de trabalho, é apresentado na figura 2.

Como demonstra o quadro 2 , o teste qui-quadrado foi significativo, e a relação qui-quadrado/graus de liberdade atingiu o valor de 2,1249, dentro do limite máximo considerado aceitável por diversos autores (Kline, 1998; Hair et al., 1999, entre outros). Tanto as demais medidas absolutas de ajuste (GFI, RMR e RMSEA) quanto as medidas comparativas (CFI, NFI e NNFI) ficaram dentro dos limites desejáveis, indicando um bom ajustamento do modelo. Isto demonstra que a retirada das relações não significativas e a inserção das covariâncias permitiram uma melhora substancial nos valores de ajustamento, quando comparado ao modelo proposto.

\section{Quadro 2 \\ Índice de ajuste do modelo de satisfação}

\begin{tabular}{|c|c|}
\hline Índice & Análise final \\
\hline Qui-quadrado & 953,996 \\
\hline Graus de liberdade & 424 \\
\hline Nível de probabilidade & 0,000 \\
\hline RFI - Relativ fit index & 0,936 \\
\hline $\mathrm{CFI}$ - Comparative fit index & 0,969 \\
\hline $\mathrm{NFI}$ - Normed fit index & 0,945 \\
\hline$|F|$ - Incremental fit index & 0,969 \\
\hline TLI - Tucker-Lewis index & 0,964 \\
\hline Pratio - Parsimony ratio & 0,855 \\
\hline PNSI - Parsimony-adjusted NFI & 0,808 \\
\hline PCFI - Parsimony-adjusted CFI & 0,828 \\
\hline RMSEA - Root mean squared error of approximation & 0,07 \\
\hline Variância extraída & 0,95 \\
\hline Confiabilidade & 0,66 \\
\hline Alpha de Cronbach & 0,909 \\
\hline AIC - Akaike information criterion & 1159,996 \\
\hline BCC - Browne-Cudeck criterion & 1189,293 \\
\hline
\end{tabular}




\section{Discussão dos resultados}

O artigo em questão visou identificar as relações funcionais de satisfação dos funcionários públicos da prefeitura de Santa Maria (RS), levando em consideração as variáveis do ambiente de trabalho, que são formadas por dois componentes: características do trabalho e contexto do trabalho.

Com base no trabalho de Wright e Davis (2003), foram avaliados oito construtos, através da técnica de equações estruturais, que interferem na satisfação dos funcionários públicos. A partir do ajuste no modelo, foram identificadas e analisadas as 11 hipóteses mencionadas na revisão. Com base nos resultados obtidos pode-se verificar que a satisfação sofre interferência direta de algumas características do trabalho, como: rotinas, especificidade e recursos humanos. E, por consequência, essas características do trabalho recebem influência direta do contexto do trabalho.

A satisfação do funcionário demonstrou ser influenciada pelas rotinas no que tange ao requerimento de habilidades, mesmos problemas, coisas novas e diferentes e rotinas previsíveis. Esses relacionamentos corroboram o posicionamento entre rotinas e satisfação mencionado nas pesquisas de Stimson e Johnson (1977). Essa mesma satisfação é influenciada pela especificidade, como mencionam os trabalhos de Daley (1986) e Ting (1996), através das variáveis: sei o que tenho que fazer, avaliação de desempenho, importância das atribuições e clareza nas responsabilidades. Concomitantemente com as relações entre rotinas e especificidade no que tange à satisfação, os recursos humanos se mostraram influenciadores da satisfação, como mostram os trabalhos de Sherman e Bohalander (1992), Roodhooft e Abbeele (2006) e Budd e Broad (1996), representados pelas variáveis: desenvolvimento da carreira, habilidades e treinamento.

Esses achados corroboram o argumento central de Wright e Davis (2003); no entanto, no caso da satisfação dos funcionários públicos da prefeitura de Santa Maria, algumas correlações foram eliminadas, como a relação entre feedback e especificidade do trabalho, feedback e RH, metas específicas organizacionais e RH. Desse modo, não se observaram algumas relações condizentes com os trabalhos de Kuvaas (2009), Andreassen (1994), Addae e Parboteeah (2008), Barnett e Bradley (2007), Wright (2001) e Jamali (2007).

Foi também confirmado que, no caso dos funcionários da prefeitura de Santa Maria, existem outras relações que influenciam indiretamente a satisfação, que não mencionadas no modelo de Wright e Davis (2003), principalmente as que dizem respeito a conflitos e especificidade, especificidade e RH e conflitos e RH.

De acordo com o resultado obtido na análise, percebe-se que a satisfação objeto da pesquisa está positivamente correlacionada com alguns dos sete construtos apresentados para avaliar o construto denominado satisfação, confirmando os estudos que colocam as características do trabalho (rotinas, especificidade do trabalho, desenvolvimento de recursos humanos e feedback) e o contexto do trabalho (conflitos nas metas organizacionais, restrições processuais e especificidade das metas organizacionais) como quesitos determinantes da satisfação do funcionário. 


\section{Considerações finais}

O presente estudo fornece como base teórica e empírica um modelo aplicado com fatores que interferem na satisfação no setor público, unificando dessa forma o entendimento das relações funcionais acerca de sete fatores relacionados à satisfação. Embora o estudo apresente uma visão parcial dos respondentes e um provável "viés" da análise dos métodos quantitativos, acredita-se que uma de suas maiores contribuições encontra-se na proposta de junção dos construtos encontrados, presentes isoladamente nos trabalhos de Stimson e Johnson (1977); Daley (1986); Ting (1996); Sherman e Bohlander (1992); Roodhooft e Abbeele (2006); Budd e Broad (1996); Kuvaas (2009); Andreassen (1994); Barnett e Bradley (2007); Snyder, Osland e Hunter (1996); Addae e Parboteeah (2008); Wright e Davis (2003); Bellou (2009); Behn (1995); Buchanan (1975); Kim (2006); Meyer (1982) e Jamali (2007), entre outros, estabelecendo, a partir desse relacionamento, a possibilidade de uma compreensão mais profunda do processo de satisfação no setor público.

Desse modo, os resultados encontrados nesta pesquisa são relevantes tanto para a academia como para a prática. Para a academia, contribui com o desenvolvimento teórico do campo de pesquisa em gestão pública. Nota-se que esse é um desafio extremamente importante para a comunidade acadêmica, pois é um campo tradicional que tem evoluído em grande escala nos últimos anos. Para a prática, o modelo será referência útil para as instituições públicas.

Novas análises são estimuladas visando superar as limitações deste artigo. Entre as limitações podem ser citadas o fato de a amostra não ser probabilística e o local de atuação se restringir à prefeitura de Santa Maria. No que se refere a estudos futuros, investigações em outros estados e países permitiriam comparar os resultados em função das especificidades locais, bem como a incorporação de outras variáveis poderia representar um avanço na construção do modelo. Desse modo, constata-se neste artigo que a satisfação no setor público é um tema evidente, especialmente pelas complexas relações existentes nas características e no contexto do trabalho.

\section{Referências}

ADDAE, Helena M.; PARBOTEEAH, Praveen K. Role stressors and organizational commitment: public sector employment in St Lucia. International Journal of Manpower, v. 29, n. 6, p. 567-582, 2008.

AGUS, Arawati; BARKER, Sunita; KANDAMPULLY, Jay. An exploratory study of service quality in the Malaysian public service sector. International Journal of Quality \& Reliability Management, v. 24, n. 2, p. 177-190, 2007.

ANDREASSEN, Tor Wallin. Satisfaction, loyalty and reputation as indicators of customer orientation in the public sector. International Journal of Public Sector Management, v. 7, n. 2, p. 16-34, 1994. 
BABAKUS, E. et al. Examining the role of organizational variables in the salesperson job satisfaction model. The Journal of Personal Selling and Sales Management, v. 16, n. 3, p. 33-42, 1996.

BALDWIN, Norman J.; FARLEY, Quinton A. Comparing the public and private sectors in the United States: a review of the empirical literature. In: FARAZMAND, Ali (Org.). Handbook of comparative and development public administration. New York: Marcel Dekker, 1991. p. 27-39.

BARNETT, Belinda Renee; BRADLEY, Lisa. The impact of organizational support for career development on career satisfaction. Career Development International, v. 12, n. 7, p. 617-636, 2007.

BELLOU, Victoria. Matching individuals and organizations: evidence from the Greek public sector. Employee Relations, v. 31, n. 5, p. 455-470, 2009.

BEHN, R.D. The big questions of public management. Public Administration Review, v. 55, n. 4, p. 313-324, 1995.

BIGNÉ, Enrique; MOLINER, Miguel A.; SÁNCHEZ, Javier. Perceived quality and satisfaction in multiservice organizations: the case of Spanish public service. Journal of Service Marketing, v. 17, n. 4, p. 420-442, 2003.

BREWR, G.A.; SELDON, S.C. Why elephants gallop?: assessing and predicting organizational performance in federal agencies. Journal of Public Administration Research and Theory, v. 10, n. 4, p. 685-711, 2000.

BRUNETTO, Yvonne; FARR-WHARTON, Rod. Using social indent theory to explain the job satisfaction of public sector employees. The International Journal of Public Sector Management, v. 15, n. 7, p. 534-551, 2002.

BUCHANAN, B. Government managers, business executives, and organizational commitment. Public Administration Review, v. 34, n. 4, p. 339-347, 1975.

BUDD, Marjori L.; BROAD, Mary L. Training and development for organizational performance. In: PERRY, James L. (Org.). Handbook of public administration. San Francisco: Jossey-Bass, 1996. p. 424-439.

DALEY, Denis M. Humanistic management and organizational success: the effect of job and work environmental characteristics on organizational effectiveness, public responsiveness and job satisfaction. Public Personnel Management, v. 15, n. 2, p. 131-142, 1986.

DESANTIS, Victor S.; DURST, Samantha L. Comparing job satisfaction among public and private sector employees. American Review of Public Administration, v. 3, n. 26, p. 327-343, 1996.

EMMERT, Mark A.; TAHER, Walled A. Public sector professionals: the effects of public sector jobs on motivation, job satisfaction and work involvement. American Review of Public Administration, v. 22 , n. 1, p. 37-48, 1992.

FU, Hsin-Pin; CHANG, Tien-Hsiang; CHAO, Pei. A collaborative model for service provision by multiple public-sector agencies. Internet Research, v. 16, n. 4, p. 365-379, 2006.

GABRIS, Gerald T. Public sector motivation as an independent variable affecting career decisions. Public Personnel Management, v. 24, n. 1, p. 33-51, 1995. 
GARVER, M.S.; MENTZER, J.T. Logistics research methods: employing structural equation modeling to test for construct validity. Journal of Business Logistics, v. 20, p. 33-57, 1999.

GIL, A.C. Como elaborar projetos de pesquisa. São Paulo: Atlas, 2002.

HAIR, J.F. et al. Fundamentos de métodos de pesquisa em administração. São Paulo: Bookman, 2006.

HAIR, J.F. et al. Analisis multivariante. 5. ed. Madrid: Prentice-Hall, 1999.

KIM, Sangmook. Public service motivation and organizational citizenship behavior in Korea. International Journal of Manpower, v. 27, n. 8, p. 722-740, 2006.

KLINE, R.B. Principles and practice of structural equation modeling. New York: The Guilford Press, 1998.

KUVAAS, Bard. A test of hypotheses derived from self-determination theory among public sector employees. Employee Relations, v. 31, n. 1, p. 39-56, 2009.

JAMALI, Dima. A study of customer satisfaction in the context of a public private partnership. International Journal of Quality \& Reliability Management, v. 24, n. 4, p. 370-385, 2007.

LEWIS, Gregory B. Pay and job satisfaction in the federal civil service. Review of Public Personnel Administration, v. 11, n. 3, p. 17-31, 1991.

LEWIS, Gregory B.; ALONSO, P. Public service motivation and job performance: evidence from the federal sector. American Review of Public Administration, v. 31, n. 4, p. 363-380, 2001.

LIU, Bangcheng; TANG, Ningyu; ZHU, Xiaomei. Public service motivation and job satisfaction in China: an investigation of generalizability and instrumentality. International Journal of Manpower, v. 29, n. 8, p. 684-699, 2008.

MAIDANI, Ebrain A. Comparative study of Herzberg's two-factor theory of job satisfaction among public and private sectors. Public Personnel Management, v. 20, n. 4, p. 441-448, 1991.

MCADAM, Rodney; REID, Renne; SAULTERS, Robbie. Sustaining quality in the UK public sector. International Journal of Quality \& Reliability Management, v. 19, n. 5, p. 581-592, 2002.

MEYER, M.W. Bureaucratic vs. profit organization. In: STAW, B.M.; CUMMINGS, L.L. (Org.). Research in organizational behavior. Greenwich: CTJAI, 1982. p. 89-126.

NUNNALY, J. Psychometric Theory. New York: McGraw-Monte, 1978.

PAINE, Frank T.; CARROLL, Stephen J.; LEETE, Burt A. Need satisfactions of managerial level personnel in a government agency. Journal of Applied Psychology, v. 50, n. 3, p. 247-249, 1966.

PEDHARZUR, Elazar J.; SCHMELKIN, Liora P. Measurement, design, and analysis: an integrated approach. New Jersey: Lawrence Erlbaum, 1991.

PERRY, James L.; PORTER, Lyman W. Factors affecting the context for motivation in public organizations. Academy of Management Review, v. 7, n. 1, p. 89-98, 1982.

PORTER, Lyman W.; MITCHELL, Vance F. Comparative study of need satisfactions in military and business hierarchies. Journal of Applied Psychology, v. 51, n. 2, p. 139-144, 1967. 
RAINEY, Hal G. Public management: recent research on the political context and managerial roles, structures and behaviors. Journal of Management, v. 15, n. 2, p. 229-250, 1989.

ROODHOOFT, Filip; ABBEELE, Alexandra Van den. Public procurement of consulting services evidence and comparison with private companies. International Journal of Public Sector Management, v. 19, n. 5, p. 490-512, 2006.

SCHUMACKER, R.E.; LOMAX, R.G. A beginner's guide to structural equation modeling. New Jersey: Lawrence Erlbaum, 1996.

SIEGLE, D. Cronbach's Alpha reliability. Disponível em: <www.delsiegle.com>. Acesso em: 16 jun. 2004.

SHERMAN, Arthur. W.; BOHLANDER, George W. Managing human resources. Cincinnati, OH: South-Western Publishing, 1992.

SNYDER, M.M.; OSLAND, J.; HUNTER, L. Public and private organizations in Latin America: a comparison of reward preferences. The International Journal of Public Sector Management, v. 9, n. 2, p.15-27, 1996.

STEEL, Brent S.; WARNER, Rebecca L. Job satisfaction among early labor force participants: unexpected outcomes in public and private sector comparisons. Review of Public Personnel Administration, v. 10, n. 3, p. 4-22, 1990.

SOLOMON, E.E. Private and public sector managers: an empirical investigation of job characteristics and organizational climate. Journal of Applied Psychology, v. 71, n. 2, p. 247-259, 1986.

STIMSON, John; JOHNSON, Thomas. Tasks, individual differences, and job satisfaction. Industrial Relations, v. 3, p. 315-322, 1977.

TING, Y. Analysis of job satisfaction of the federal white-collar work force: findings from the survey of federal employees. American Review of Public Administration, v. 26, n. 4, p. 439-456, 1996.

WRIGHT, Bradley E. Public sector work motivation: review of current literature and a revised conceptual model. Journal of Public Administration and Theory, v. 11, n. 4, p. 559-586, 2001.

WRIGHT, Bradley E.; DAVIS, Braian S. Job satisfaction in the public sector: the role of the work environment. The American Review of Public Administration, v. 33, n. 7, 2003.

Wagner Junior Ladeira é doutorando do Programa de Pós-Graduação em Administração da Universidade Federal do Rio Grande do Sul (UFRGS). E-mail: wjladeira@ea.ufrgs.br.

Igor Bernardi Sonza é doutorando do Programa de Pós-Graduação em Administração da UFRGS. E-mail: igorsonza@gmail.com.

Roberto Sarquis Berte é gerente do Núcleo de Educação Profissional da Senac Technology School. Email: rsberte@senacrs.com.br. 